Rabaska

Revue d'ethnologie de l'Amérique française

\title{
Maison des arts de la parole
}

\section{Sophie Jeukens}

Volume 11, 2013

URI : https://id.erudit.org/iderudit/1018566ar

DOI : https://doi.org/10.7202/1018566ar

Aller au sommaire du numéro

Éditeur(s)

Société québécoise d'ethnologie

ISSN

1703-7433 (imprimé)

1916-7350 (numérique)

Découvrir la revue

Citer ce document

Jeukens, S. (2013). Maison des arts de la parole. Rabaska, 11, 299-300.

https://doi.org/10.7202/1018566ar

Ce document est protégé par la loi sur le droit d'auteur. L'utilisation des services d'Érudit (y compris la reproduction) est assujettie à sa politique d'utilisation que vous pouvez consulter en ligne.

https://apropos.erudit.org/fr/usagers/politique-dutilisation/
Cet article est diffusé et préservé par Érudit.

Érudit est un consortium interuniversitaire sans but lucratif composé de l’Université de Montréal, l'Université Laval et l'Université du Québec à Montréal. Il a pour mission la promotion et la valorisation de la recherche. https://www.erudit.org/fr/ 


\title{
Maison des arts de la parole
}

138 , rue Wellington Nord, $2^{\mathrm{e}}$ étage

Sherbrooke (Québec)

J1H 5C5

\author{
Téléphone : (819) 566-6996 \\ Télécopieur : (819) 780-8972
}

Courriel : info@maisondesartsdelaparole.com

Toile : www.maisondesartsdelaparole.com

\section{QuAND LA PAROLE NOUS HABITE, ON S'EN FAIT UNE MAISON}

Ce fut une année plus grande que nature, dans le petit monde de Productions Littorale, qui troquait, il y a un an à peine, son nom chargé de dix ans d'histoire pour en prendre un nouveau : celui de Maison des arts de la parole. Certes, nous n'en sommes pas encore à habiter notre propre édifice, mais l'esprit chaleureux et convivial avec lequel nous accueillons, chaque année, des artistes de partout dans le monde mérite bien l'appellation de maison, aussi abstraite soit-elle. Sans compter que cette maison, c'est aussi celle qui fut l'espace privilégié des veillées de conte d'antan. Comme un clin d'œil à l'histoire d'une littérature orale dont nous retissons aujourd'hui le fil millénaire, tout en visitant ses plus contemporaines expressions.

\section{LES JOURS SONT CONTÉS}

L'année s'est ouverte sous le signe de la fête : celle du festival de conte Les jours sont contés, devenu en octobre dernier le premier festival de conte au Québec à célébrer sa vingtième édition. Pour l'occasion, c'est une programmation d'une qualité exceptionnelle, toute en coups de cœur, qui a été échafaudée, regroupant des artistes d'ici (Québec, Ontario) et d'ailleurs (France, Belgique, Espagne, Portugal, Argentine et Liban) dont plusieurs nous font l'honneur de fréquenter le festival depuis plusieurs années. Fidèle à ses habitudes, l'événement s'est étendu à travers toute la région estrienne, de Coaticook à Richmond, de Saint-Camille à Frelighsburg, et, pour la première fois, jusque dans la grande ville de Montréal, où une dizaine de spectacles ont été présentés gratuitement dans les salles du réseau Accès Culture.

En plus des quelque 79 spectacles proposés, nombre de projets spéciaux ont été mis sur pied : une parade d'ouverture guidée en chansons par le conteur-musicien Michel Hindenoch, une exposition de photos grappillées au fil de ces vingt dernières années, un journal quotidien - produit chaque jour aux petites heures du matin -, ainsi que le lancement d'un disque compact et d'une bande dessinée mettant en lumière le travail de quelques-uns des artistes invités de cette édition hors normes. Et parce que nous croyons en la nécessité de mener un travail théorique rigoureux en regard du conte, art à la fois millénaire et émergent, nous avons également profité de ce moment de rassemblement pour orchestrer une activité de réflexion, regroupant théoriciens et praticiens autour d'une question : celle du travail du conte. Une initiative qui, espérons-le, saura contribuer, à sa façon, à la reconnaissance de l'art du conte et à son développement au Québec.

Si nous souhaitions avant tout faire de cet anniversaire un moment mémorable, dont la couverture médiatique et la grande qualité artistique permettraient de familiariser de nouveaux publics avec cet art méconnu qu'est le conte, il va sans dire que l'augmentation de plus de $60 \%$ de notre assistance a su dépasser nos ambitieuses espérances. Pas de répit pour l'équipe après ce festival endiablé. 


\section{Autres ACTIVITÉs}

En mars, en collaboration avec le Cercle des conteurs des Cantons de l'Est, nous avons proposé aux Sherbrookois une dizaine d'activités, spectacles pour petites et grandes oreilles et ateliers de contes et comptines, à l'occasion de la Journée mondiale du conte - qui, depuis longtemps déjà, s'étire par chez nous sur une semaine entière !

Nous avons également accueilli, au printemps, deux formatrices d'expérience venues d'outre-mer partager leur savoir aux artistes d'ici. Alors que Françoise Diep a proposé un stage sur l'art de conter aux tout-petits à un groupe de conteurs et conteuses émergents et de professionnelles de la petite enfance - dont plusieurs découvraient pour la première fois le conte oral -, Christine Andrien a permis à douze conteurs de se mouiller pour trois jours à la pédagogie de l'École internationale du conte de Bruxelles, dont elle est codirectrice. Une rencontre qui a su alimenter nos réflexions quant au développement d'un véritable programme de formation en conte en terres québécoises !

La saison estivale, pour sa part, a été marquée par un projet tout spécial, grâce auquel les passagers du Grand Cru, bateau de croisière touristique naviguant sur les eaux du lac Memphrémagog de juin à octobre, ont eu l'occasion d'entendre un conte sur fond de trame historique, écrit sur mesure et livré tour à tour par cinq artistes de la région, au cours de cent cinq représentations.

\section{ENTRE CONTINUITÉ ET NOUVEAUTÉ}

Dotée d'un nouveau nom et d'une image graphique entièrement revisitée, la Maison des arts de la parole souhaite, plus que jamais, explorer les diverses formes que peut prendre la parole partagée, sur un chemin qui serpente entre continuité et nouveauté. L'organisme entre d'ailleurs dans une année charnière de son histoire : sa fondatrice, Petronella Van Dijk, en ayant successivement cédé la direction générale et la direction artistique, ce sont deux jeunes artistes de la région, Marie Lupien-Durocher et Sophie Jeukens, qui reprennent fièrement le flambeau, fortes de vingt ans d'histoire, mais également remplies d'idées nouvelles.

Alors que la poésie orale et performée se taille doucement une place au creux de notre programmation, qui compte désormais un deuxième festival - le Festival $d u$ texte court, axé sur les pratiques poétiques et performatives brèves -, le désir de maintenir, dans notre monde, des espaces de rencontre humaine, des moments de partage autour de cette tradition millénaire et universelle qu'est la littérature orale, continue de former le cœur de notre mission. 